\title{
Analytische Philosophie
}

\section{Peter Bieris Frage nach der richtigen Art, Philosophie zu betreiben}

\section{Von ANSGAR BECKERMANN (Bielefeld)}

In Ferdinand Fellmanns Orientierung Philosophie findet sich die folgende launige Charakterisierung der Analytischen Philosophie:

„In philosophischen Seminaren erkennt man [sprachanalytische Philosophen] auf den ersten Blick an ihrem forschen Auftreten und der Verve, mit der sie ganze Tafeln vollschreiben. Was sie schreiben, sind in der Regel triviale Beispielsätze sowie logische Zeichen, deren virtuose Beherrschung alle Erstsemester beeindruckt. Daß sie von der Geschichte der Philosophie vor Bertrand Russell und George Edward Moore [...] augenscheinlich wenig Notiz nehmen und sich auf Wittgenstein, Willard Van Orman Quine und Georg Henrik von Wright als Referenzautoren beziehen, kommt ihrem Modernitätsbewußtsein zugute. [...] [Alle sprachanalytischen Philosophen] vereint [...] ein pragmatisch und realistisch ausgerichtetes Denken, das seinen amerikanischen Ursprung nicht verleugnen kann. Das macht den Charme dieser Gruppe von Philosophen aus, die rein problemorientiert denken. Dagegen sind sie den humanistisch gebildeten Geistern höchst suspekt, wenn nicht gar unsympathisch. Kann man doch oft genug erleben, daß die analytischen Philosophen formal bombastische Apparaturen für Einsichten einsetzen, die aus der Tradition längst vertraut sind.“ (Fellmann 1998, 95)

Diese Karikatur könnte man getrost beiseite legen, gäbe es nicht auch gewichtigere Stimmen, die dieser Art des Philosophierens inzwischen ebenfalls eher kritisch gegenüberstehen. In seinem Aufsatz Was bleibt von der Analytischen Philosophie?, der sich wie das Dokument einer enttäuschten Liebe liest, listet Peter Bieri - mit seltsamem publizistischen Echo - alles auf, was ihm an der Analytischen Philosophie missfällt, um am Ende dafür zu plädieren, Die beiden nachfolgenden Beiträge reagieren auf die Kritik der analytischen Philosophie, die Peter Bieri im Heft 3/2007 dieser Zeitschrift vorgelegt hat.

Vgl. zu diesem Aufsatz auch Beckermann (2004).

Geyer (2007). 
dass es weder analytische noch kontinentale Philosophie geben sollte, sondern einfach nur Philosophie.

Erstaunlich an Bieris Aufsatz ist, dass er auf die Anfänge der Analytischen Philosophie - auf die Zeiten, in denen noch ziemlich klar war, was man wollte und was nicht - nur beiläufig eingeht. Dabei sind gerade diese Anfänge für ein Verständnis dessen, was Analytische Philosophie ausmacht (oder ausmachte), enorm wichtig. Werfen wir deshalb zunächst einen Blick zurück.

Ich halte es für keine gute Idee, schon Frege, Moore und Russell (sozusagen rückwirkend) zu Analytischen Philosophen zu erklären. ${ }^{3}$ Die Analytische Philosophie beginnt mit Wittgenstein und dem Wiener Kreis, und ihr Grundimpetus ist antimetaphysisch, ja sogar antiphilosophisch. Sinnvoll sind allein Aussagen, die sich durch Erfahrung bestätigen oder widerlegen lassen. Die einzigen legitimen Wissenschaften sind die Erfahrungswissenschaften. In ihrem Manifest Wissenschaftliche Weltauffassung - der Wiener Kreis schreiben Carnap, Hahn und Neurath: ,[E]s gibt nur Erfahrungserkenntnis [...]. Hiermit ist die Grenze für den Inhalt legitimer Wissenschaft gezogen." Schon im Tractatus ist diese Auffassung ganz deutlich: Sinnvoll sind nur Elementarsätze und logische Verknüpfungen von Elementarsätzen. Logische und mathematische Sätze sind Grenzfälle; sie sagen nichts über die Welt; sie sind daher zwar nicht unsinnig, aber sinnlos. Erst später findet sich in der Analytischen Philosophie ein Ort fü Logik und Mathematik. Man beginnt, zwei Arten sinnvoller Aussagen zu unterscheiden: analytische Aussagen und Aussagen, die sich durch Erfahrung bestätigen oder widerlegen lassen, und rettet der Logik und der Mathematik einen Platz, indem man ihre Aussagen für analy tisch erklärt. Doch dabei bleibt die Frage: Und was ist mit der Philosophie? Die Antwort, die man allgemein findet, lautet: Der Philosophie bleibt nur die Sprachanalyse. Aber wie pass Sprachanalyse selbst in den Rahmen der legitimen Wissenschaften? Im Tractatus gibt Wittgenstein eine radikale Antwort: Auch Sprachanalyse ist eigentlich nicht möglich. Philosophie möchte über Sprache und das Verhältnis von Sprache und Welt reden; aber genau das geh nicht. Reden kann man nur über Gegenstände - mit Sätzen, die Sachverhalte ausdrücken, das heißt die besagen, dass die Gegenstände so-und-so verkettet sind. Über Sachverhalte reden kann man nicht, und damit auch nicht über Sätze. Zwar kann man Sprache verwenden, un den Hörer/Leser dazu zu bringen, bestimmte Dinge richtig zu sehen, über die man eigentlich nicht reden kann; aber diese Sprachverwendung ist streng genommen sinnlos. Das dem Paradoxon, dass man die Leiter - diese sinnlosen Sätze - wegwerfen muss, nachdem man gesehen hat, was sie uns sehen lernen sollte.

Neurath scheint ein anderes Verständnis von Sprachanalyse gehabt zu haben; für ihn wa sie eine ganz normale empirische Wissenschaft. Aber bei den meisten analytischen Philosophen dieser Zeit findet sich keine klare Antwort auf die Frage nach dem Status von Sprachanalyse - am ehesten noch die These, Sprachanalyse sei keine Theorie, sondern eine Tätigkeit, daher stelle sich die Frage nach ihrem Status als Wissenschaft gar nicht. Klar ist allerdings, was mit Sprachanalyse erreicht werden soll. Mit ihrer Hilfe soll erstens gezeigt werden, dass es nur die zwei genannten Arten von sinnvollen Sätzen gibt; und es soll zweitens gezeigt werden, dass die Sätze der klassischen Philosophie/Metaphysik letzten Endes alle sinnlos sind. In seinem Aufsatz Überwindung der Metaphysik durch logische Analyse der Sprache schreib Carnap klar und eindeutig, sein Ziel sei, ,auf die Frage nach der Gültigkeit und Berechtigung der Metaphysik eine neue und schärfere Antwort zu geben [...] Auf dem Gebiet der Metaphysik [...] führt die logische Analyse zu dem negativen Ergebnis, dass die vorgeblichen Sätze dieses Gebiets gänzlich sinnlos sind“" (Carnap 1931, 219 f.).

Natürlich kann man das mit guten Gründen auch anders sehen; vgl. etwa Glock (2008).
Wie für Carnap, Hahn und Neurath, so ist, wie wir schon gesehen haben, auch für den Wittgenstein des Tractatus „Metaphysik“ ein Name für die illegitime traditionelle Philosophie. Legitime Philosophie ist für ihn Kritik der Sprache.

„The only legitimate task of philosophy is analytic and elucidatory. It neither aims at the discovery of new truths, nor shares the piecemeal methods of the sciences. For there are no ,philosophical propositions'. Philosophy, unlike science, is not a body of doctrine, but an activity of clarifying non-philosophical propositions through logical analysis [...]." (Glock 1996, 294)

Diese Auffassung von Philosophie hat Wittgenstein in seinen späteren Schriften zwar weiter entwickelt; aber die Grundlinie blieb dieselbe - die Ablehnung der Idee, es könne so etwas wie eine substanzielle Philosophie geben. Und diese Idee findet sich in unterschiedlicher Form auch bei anderen Vertretern der Philosophie der normalen Sprache wie etwa Ryle.

Wenn man die metaphysik- und philosophiekritische Einstellung, die alle Vertreter der aufstrebenden Analytischen Philosophie einte, mit dem vergleicht, was heute unter dem Namen „Analytische Philosophie“ betrieben wird, kommt man nicht umhin, zuzugeben, dass sich vieles grundlegend verändert hat. Und dies ist umso verblüffender, als es, oberflächlich gesehen, gar keinen erkennbaren Bruch in der Entwicklung der Analytischen Philosophie gegeben zu haben scheint. Mehr oder weniger unmerklich, darauf weist Bieri völlig zu Recht hin, kehrten die traditionellen Themen der Philosophie zurück bis die überkommene Philosophie eine vollständige Wiederauferstehung feiern konnte. Wie war das möglich? Wie konnte es zu solch einem grundstürzenden Umschwung kommen?

In der Regel werden hier zwei Namen und zwei Werke angeführt, die den Umschwung vielleicht nicht wirklich einleiteten, aber doch ein deutliches Zeichen dafür waren, dass etwas in Gang gekommen war - Quines Two Dogmas of Empiricism von 1951 und Peter F. Strawsons Individuals von 1959. Dies trifft nur zum Teil zu. Wenn ich es richtig sehe, war der wichtigste Grund des Umbruchs das Scheitern des empiristischen Sinnkriteriums.

Schon Popper hatte sich geweigert, von einem „Sinnkriterium“ zu sprechen, und vorgeschlagen, stattdessen den Ausdruck „Abgrenzungskriterium“ zu verwenden. Sätze, die sich empirisch falsifizieren lassen, sind wissenschaftliche Sätze; Sätze, bei denen das nicht der Fall ist, gehören nach Popper zwar nicht in den Bereich der Wissenschaften, sind deshalb aber noch lange nicht sinnlos. Doch weniger Poppers Vorschlag zur Güte als vielmehr die Erkenntnis, dass auch zentrale wissenschaftliche Ausdrücke wie „Elektron" oder sogar „Masse“ den strengen Anforderungen des empiristischen Sinnkriteriums nicht genügen, führte dazu, dass dieses Kriterium im Laufe der vierziger und fünfziger Jahre des 20. Jahrhunderts Schritt für Schritt aufgegeben wurde. Doch damit war der Analytischen Philosophie ein zentrales Werkzeug zur Destruktion der traditionellen Philosophie abhanden gekommen. Wenn Termini wie „Elektron“ und „Masse“ keinen klaren empirischen Gehalt haben, warum sollte man das von Ausdrücken wie ,Prinzip“ oder ,Gott“" erwarten? Wenn Sätze wie ,Elektronen haben vine A ist der Schöpfer der Welt" sinnlos sein? Der Fall des empiristischen Sinnkriteriums war, wenn man mir dieses Bild verzeiht, die Einbruchstelle, durch die zunächst einmal die traditionelle philosophische Terminologie in die Analytische Philosophie zurückkehren konnte. Und in 
deren Gefolge kamen auch die Probleme der traditionellen Philosophie zurück, und zwar in rasantem Tempo und ohne dass dies großes Aufsehen hervorrief.

Am verblüffendsten war vielleicht die Wiederkehr der normativen Ethik. Nach vielen Jahren, in denen sich ethische Überlegungen - der Idee folgend, Philosophie müsse sich au Sprachanalyse beschränken - allein auf Metaethik beschränkt hatten, war es, hauptsächlich wohl angestoßen durch John Rawls' A Theory of Justice von 1971, plötzlich wieder möglich, über Freiheit und Gerechtigkeit zu reden, über den Status ungeborenen Lebens und über den Umgang mit Sterbenden. Schon in den siebziger Jahren des 20. Jahrhunderts blieb somit nur die Feststellung: Das Projekt der Abschaffung der Metaphysik durch logische Analyse der Sprache war grandios gescheitert.

Rückblickend kann man also sagen, dass die Analytische Philosophie ursprünglich durch zumindest eine der folgenden drei Thesen gekennzeichnet war:

(1) Ziel der Philosophie ist die Überwindung der Metaphysik durch Sprachanalyse.

(2) Die einzige (legitime) Aufgabe der Philosophie ist die Analyse der (Alltags- oder Wissenschafts-)Sprache.

(3) Die einzige Methode, die der Philosophie zur Verfügung steht, ist die Methode der Sprachanalyse.

Aber spätesten 1975 gab es kaum noch jemanden, der auch nur eine dieser Thesen unterschrieben hätte. Mit anderen Worten: Die Analytische Philosophie in dem Sinne, in dem dieser Ausdruck in der ersten Hälfte des 20. Jahrhunderts verstanden wurde, war passé. Die traditionelle Analytische Philosophie war, lautlos, untergegangen.

III.

Gibt es trotzdem etwas, was auch heute noch alle analytischen Philosophen eint und von anderen Philosophen abgrenzt? Bieri spricht von einer „Reihe von gedanklichen Elementen, auf die man immer wieder trifft, wenn Autoren, die sich als analytische Philosophen verstehen, ihr Selbstbild zum Ausdruck bringen. [...] Diese ideologischen Elemente sind das einzige Material, an dem man sich orientieren kann.“ (Bieri 2007, 333) Zu diesen gedanklichen Elementen zählt in seinen Augen:

die Auffassung, dass Philosophie ein „normales“ Fach ist;

- das besondere Gewicht, das auf begriffliche Klarheit - im Sinne von Exaktheit (formalsprachlicher Zweig) oder Ubersichtlichkeit (normalsprachlicher Zweig) gelegt wird;

die besondere Rolle der formalen Logik;

- die besondere Rolle des ,semantic ascent".

Ich werde auf einige dieser Punkte zurückkommen. In Bieris Aufzählung werden aber einige inhaltliche Punkte außer Acht gelassen, die zumindest für eine gewisse Zeit für das Selbstverständnis der Analytischen Philosophie noch zentraler waren. In erster Linie denke ich an die große Wissenschaftsnähe der Analytischen Philosophie und ihren ausgeprägten Antimentalis mus. Das Erste mag für den normalsprachlichen Zweig der Analytischen Philosophie wenige zutreffen. Doch wir hatten schon gesehen, dass zumindest zu Beginn des formalsprachlichen
Zweiges die Erfahrungswissenschaften als einzig legitime Wissenschaften galten. Auch nachdem die Idee eines Monopols der Erfahrungswissenschaften aufgegeben wurde, änderte sich nichts am herausgehobenen Status, den viele analytische Philosophen den empirischen Wissenschaften einräumten. Das zeigt sich besonders bei Quine, aber auch bei Sellars, wenn er bei nicht lösbaren Konflikten zwischen dem „manifest“ und dem „scientific image“ der Welt Letzterem den Vorrang gibt. ${ }^{4}$ Und ganz deutlich zeigt es sich bei David Lewis. Mit Bezug auf die Mathematik schreibt Lewis in Parts of Classes:

Mathematics is an established, going concern. Philosophy is as shaky as can be. To reject mathematics for philosophical reasons would be absurd. [...] How would you like the job of telling the mathematicians that they must change their ways, and abjure countless errors, now that philosophy has discovered that there are no classes? Can you tell them, with a straight face, to follow philosophical argument wherever it may lead? If they challenge your credentials, will you boast of philosophy's other great discoveries: that motion is impossible, that a Being than which no greater can be conceived cannot be conceived not to exist, that it is unthinkable that there is anything outside the mind, that time is unreal, that no theory has ever been made at all probable by evidence [...], and so on, and on, ad nauseam? Not me!“(Lewis 1991, 58 f.)

Ähnlich dachte Lewis über die empirischen Wissenschaften, besonders die Physik. Wenn überhaupt etwas, dann informiert uns die Physik darüber, wie die Welt beschaffen ist. Wenn die Physik uns sagt, dass es Teilchen und Felder gibt, dann gibt es keinen philosophischen Grund, daran zu zweifeln.

Andere sehen in wissenschaftlichen Theorien nur hilfreiche Instrumente zur Vorhersage von Beobachtungen oder zur Konstruktion von Geräten. Für Lewis kommt das nicht in Frage: Wir haben zwar keine Garantie, dass unsere besten Theorien wahr sind - wir können nicht einmal beweisen, dass die Welt nicht erst vor fünf Minuten entstand -, aber solange wir keine konkreten Verdachtsmomente gegen sie haben, sollte uns das nicht davon abhalten, unseren Theorien zu vertrauen und zu glauben, dass sie die Welt in etwa, wenn auch sicher nicht ganz, korrekt erfassen. Woran sollten wir sonst glauben, wenn nicht an unsere besten Theorien: an schlechtere Theorien? Oder an gar nichts?" (Schwarz 2008,8 f.)

Philosophische Überlegungen sind demgegenüber prinzipiell zweitrangig. Wenn sie den Ergebnissen etablierter Wissenschaften widersprechen, dann hat für Lewis meist die Philosophie klein beizugeben. Philosophische Überlegungen sind in seinen Augen in der Regel fragwürdiger als wissenschaftliche Ergebnisse. Diese Tendenz zum wissenschaftlichen Realismus, die anatischen Philosophen oft den Vorwurf des Szientismus eingetragen hat, scheint mir Tat eine wesentliche Gemeinsamkeit der meisten analytischen Philosophen zu sein - ebenso wie die damit zusammenhängende Tendenz zum Naturalismus.

Die These von der Einheitswissenschaft gehörte zu den expliziten Programmpunkten des Wiener Kreises. Und auch nach der Erkenntnis, dass die Wissenschaften nicht in dem Sinne eine Einheit bilden, wie man sich das ursprünglich vorgestellt hatte, änderte sich nichts an

${ }^{4},,[\mathrm{I}] \mathrm{n}$ the dimension of describing and explaining the world, science is the measure of all things, of what is that it is, and of what is not that it is not." (Sellars 1963, 173)

"If current analytic philosophy can be said to have a philosophical ideology, it is, unquestionably, naturalism." (Kim 2003,84) 
den naturalistischen Grundüberzeugungen der meisten analytischen Philosophen. Vielleich ist es unpassend, die Vertreter des normalsprachlichen Zweiges der Analytischen Philosophie als ,naturalistisch“" zu bezeichnen; aber der antimentalistische Grundzug bei Wittgenstein und Ryle ist unübersehbar.

Mir scheint, dass der Vorrang der empirischen Wissenschaften und die Tendenz zum Naturalismus eine wesentliche Differenz zwischen Analytischen Philosophen und ihren Gegnern markieren (markierten?). Nehmen wir noch einmal das Zitat von Ferdinand Fellmann. ,[Alle sprachanalytischen Philosophen] vereint [...] ein pragmatisch und realistisch ausgerichtetes Denken, das seinen amerikanischen Ursprung nicht verleugnen kann. Das macht den Charme dieser Gruppe von Philosophen aus, die rein problemorientiert denken. Dagegen sind sie den humanistisch gebildeten Geistern höchst suspekt, wenn nicht gar unsympathisch." Fellmann bemerkt hier etwas ganz Richtiges, nämlich die ablehnende Haltung vieler deutscher und europäischer Philosophen der Analytischen Philosophie gegenüber. Worauf beruht diese Aversion? Nur auf der vermeintlichen Tatsache, dass analytische Philosophen über zu geringe Aversion? Nur auf der vermeintlichen Tatsache, dass analytische Philosophen über zu geringe
Kenntnisse der Philosophiegeschichte verfügen? Oder darauf, dass sie „formal bombastische Apparaturen für Einsichten einsetzen, die aus der Tradition längst vertraut sind“? Nein, mir scheint, es sind der Szientismus und der Naturalismus der Analytischen Philosophie, gegen die an Kant, Hegel, Schopenhauer, Nietzsche oder Heidegger orientierte Philosophen eine unüberwindliche Abneigung empfinden.

Damit ergibt sich aber auch ein erneuter Grund zu der Frage, ob es die Analytische Philosophie überhaupt noch gibt. Denn es hat den Anschein, als ob der Analytischen Philosophie in den letzten Jahren nicht nur ihr antimetaphysischer, sondern auch ihr szientistischer und ih naturalistischer Impetus verloren gegangen sind. Sind Plantinga und Swinburne, sind Lowe und Galen Strawson, sind Uwe Meixner und Barry Smith noch analytische Philosophen? Wenn man diese Frage mit Ja beantwortet, nimmt man Abschied von vielem, was zumindest der Mehrheit der analytischen Philosophen einmal gemeinsam war. Gibt es trotzdem noch etwas, was sie alle eint und von anderen Philosophen abgrenzt?

\section{IV.}

Kommen wir zurück zu den schon am Anfang des letzten Abschnitts angesprochenen ideologischen Elementen, die Bieri zufolge das Selbstbild der Analytischen Philosophie ausmachen. Für Bieri ist dieses Selbstbild zugleich ein Stein des Anstoßes; in seinen Augen sollte Analytische Philosophie, sollte Philosophie überhaupt, in vielfacher Hinsicht nicht so sein, wie sie sich selbst versteht. Unter anderem kritisiert Bieri, dass die formale Logik eine zu große Rolle in der Analytischen Philosophie spiele. (Auf diesen Punkt spielt ja auch Fellmann an und dieser Punkt erklärt, warum es durchaus sinnvoll ist, Plantinga und Meixner für analy tische Philosophen zu halten.) Bieri schreibt: „Auffällig ist, wie wenig [die neuen logischen Systeme] uns bei substanziellen philosophischen Fragen genützt haben." Noch schlimmer: Die starke Orientierung an Entwicklungen der formalen Logik habe bewirkt,

,dass Philosophie manchmal auszusehen begann wie Schach oder Hobbymathematik und "dass die Fragestellungen wie Logeleien klangen. Studenten wurden dadurch abgeschreckt. Nicht, weil sie die formalen Dinge nicht verstanden. Der Grund war ernster: Sie erkannten sich mit ihrer Motivation, die sie zur Philosophie gebracht hatte, in den Texten nicht mehr wieder. Mir selbst ging es nicht anders. Man war auf die Suche nach einem umfassenden
Verständnis der Welt und unserer Stellung in ihr gegangen und fand sich plötzlich in den Händen partikularistischer Techniker." (337)

Diese Diagnose halte ich für zutiefst ungerecht; und, ehrlich gesagt, ich bin noch nie einem analytischen Philosophen begegnet, der versuchte, seine Gegner mit logischen Tricksereien aufs Kreuz zu legen. ${ }^{6}$ Sicher laufen logische Formalismen manchmal ins Leere. Aber wenn wir zunächst an die Anfänge der Analytischen Philosophie denken, ist die hilfreiche Rolle der auf Frege und Russell zurückgehenden neuen Logik unbestreitbar. Ohne Freges ,Entdeckung von Quantoren und mehrstelligen Prädikaten und ohne seine Idee, dass es sich bei Quantoren um Ausdrücke für Begriffe zweiter Stufe handelt, sowie die sich aus dieser Idee ergebende neue Formelsprache wären uns viele logische Zusammenhänge bei weitem nicht so klar, wie sie es heute sind. Man denke nur an Probleme wie die Stellung und den Bereich von Quantoren, oder die Stellung von Negationszeichen und Ausdrücken wie „notwendig“ und "möglich" Der Existenzquantor ist uns so geläufig dass wir gar nicht mehr sehen, dass erst durch seine Einführung ein neues Verständnis des Wortes ,existieren“ möglich wurde - ein Verständnis, das dann später durch die freien Logiken wieder problematisiert wurde. Bieri aber behauptet:

„Was hat uns die epistemische Logik bei der Frage nach den Grenzen unseres Wissens, beim Verständnis der skeptischen Herausforderung oder bei der Analyse von Selbsterkenntnis genützt? Das Verständnis unserer Modalbegriffe ist für die Philosophie von allergrößter Bedeutung. Findet man jedoch das, worauf es ankommt, in den modalen Kalkülen? Und wissen wir seit der Formalisierung kontrafaktischer Kalküle wirklich besser, was ein Naturgesetz ist?" (Ebd.)

Bieris Urteil ist hier äußerst einseitig, und er unterschlägt, dass Logik nicht nur aus Kalkülen besteht. Will er wirklich bestreiten, dass die Mögliche-Welten-Semantik unser Verständnis der Modalbegriffe entscheidend vorangebracht hat? Will er bestreiten, dass diese Semantik für eine Vielzahl philosophischer Probleme von größter Bedeutung ist? In meinen Augen ist offensichtlich, dass die mit der Mögliche-Welten-Semantik verbundenen neuen Ausdrucksweisen bei vielen Problemen eine klarere Formulierung ermöglichen. Das gilt für so verschiedene Probleme wie den ontologischen Gottesbeweis, die Identitätstheorie in der Philosophie des Geistes, das Problem der Kausalität. Wer bestreitet, dass sich durch Lewis' Counterfactuals unser Verständnis der Wahrheitsbedingungen kontrafaktischer Konditionalsätze entscheidend verbessert hat, der bestreitet das Offensichtliche.

Auch die Suche nach expliziten Definitionen, die Bieri ebenfalls kritisch beurteilt, hat in meinen Augen, obwohl oder vielleicht gerade weil sie in vielen Fällen erfolglos war, viel Interessantes zu Tage gefördert. Die Diskussion um den Begriff des Wissens sehen viele (auch Bieri) inzwischen als ein mehr oder weniger nutzloses Austauschen von Beispielen und Gegenbeispielen. Das mag so sein. Trotzdem, Gettiers Entdeckung von Fällen, in denen wir nicht von Wissen sprechen würden, obwohl die drei Bedingungen des traditionellen Wissensbegriffs - Wissen $=$ gerechtfertigte, wahre Überzeugung - alle erfüllt sind, war ein wichtiges Ergebnis. Und die an diese Entdeckung anschließende Diskussion hat die Erkenntnistheorie ein erhebliches Stück weiter gebracht. Unter anderem dadurch, dass nun plötzlich die Bedeutung verlässlicher Mechanismen der Überzeugungsgewinnung zum ersten Mal richtig gewürdigt wurde. Ganz ohne Zweifel wurden in dieser Diskussion alte Zusammenhänge klarer und

\footnotetext{
Vgl. Geyer (2007)
} 
neue Zusammenhänge überhaupt erst sichtbar gemacht, sodass wir das gesamte Feld heute sehr viel besser verstehen als früher.

Dies ist ganz generell ein nicht zu unterschätzender Fortschritt, den die Philosophie durch die Anwendung analytischer Methoden in den letzten hundert Jahren gemacht hat. Ob wir in der Religionsphilosophie die so genannten Gottesbeweise oder die Struktur des Problems des Übels nehmen, ob wir in der Erkenntnistheorie das Problem des Skeptizismus oder den Begriff der Rechtfertigung nehmen, ob wir in der Sprachphilosophie die Frage nehmen, wie sprachliche Ausdrücke zu ihren Bedeutungen kommen, oder schließlich in der Philosophie des Geistes die Frage nach der Naturalisierbarkeit des Mentalen, ganz generell verstehen wi diese Probleme heute sehr viel besser als vor 100 Jahren

Gerade bei dem letzten Punkt wird manchmal allerdings eingewandt, dass sich die Diskussion heute so sehr in immer raffiniertere Detaildiskussionen verstrickt hat, dass das Ganze kaum noch anders als ein Glasperlenspiel gesehen werden kann. Mir scheint das weitgehend falsch. Sicher kann nicht jeder den Debatten um eine zweidimensionale Semantik etwas abgewinnen. Aber sie stellen einen ganz wesentlichen Beitrag nicht nur zur Sprachphilosophie, sondern auch zur Philosophie des Geistes dar. Und was die Sprachphilosophie angeht: Möchte jemand bestreiten, dass diese Disziplin ohne die Beiträge von Frege, Russell, Wittgenstein, Austin, Quine, Kripke, Davidson und Kaplan heute sehr viel ärmer wäre? (Von wie vielen Beiträgen außerhalb der Analytischen Philosophie im 20. Jahrhundert kann man Ähnliches sagen?) Zurück zur Philosophie des Geistes: Mir scheint, dass man um den Begriff der Supervenienz tatsächlich nicht herumkommt, wenn es um das Verständnis des Verhältnisses von Geist und Körper geht. Auch hier hat also ein eher technischer Begriff einen enormen Fortschritt im Verständnis der Sache ermöglicht.

Der Kern des Unbehagens Bieris an der Analytischen Philosophie offenbart sich meiner Meinung nach allerdings vor allem in seiner Klage, dass analytische Philosophen versucht hätten, die Philosophie zu professionalisieren, sie zu einem „,normalen“ Fach unter anderen werden zu lassen.

„Die analytischen Philosophen haben versucht, die Philosophie zu professionalisieren. Sie haben versucht, sie nach dem Modell einer Expertenkultur zu betrachten - nach dem Modell einer Aktivität also, die sich niederschlägt in Fachzeitschriften, Fachlexika, Fachkongressen und Gesellschaften, in denen sich die Fachkollegen zusammenschließen, sowie in einer weitläufigen, ausgefeilten Fachsprache, die außerhalb der Mauern der Akademie kein Mensch versteht. Auch die Rhetorik von ,philosophischer Forschung ' und von philosophischen ,Forschungsprojekten“ gehört dazu."“(333 f.)

Der Versuch der Professionalisierung muss nach Bieri aber scheitern. Denn Fächer seien durch „einen Themenbereich und eine Methode“ (334) definiert. Und beides gäbe es in de Philosophie nicht: In der Philosophie gäbe es weder eine „stabile fachliche Matrix“ noch ein „,methodologisches Repertoire, das einen auf überschaubare, nachvollziehbare Weise von Thema zu Thema, von Problem zu Problem führte“ (335). Das mag schon sein. Aber gilt dies nicht auch für alle anderen Wissenschaften? Welche ,stabile fachliche Matrix“ würde etwa die verschiedenen Bereiche der Physik oder der Biologie zusammenhalten? Gibt es in diesen Wissenschaften wirklich ein „methodologisches Repertoire, das einen auf überschaubare, nachvollziehbare Weise“ von der Quantentheorie zur Hydrodynamik oder von der Molekularbiologie zur Verhaltensforschung führen würde? Meines Wissens ist das nicht so. Es wäre auch zu viel verlangt. Fächer bilden keine monolithischen Strukturen. Und sie werden in der Regel keineswegs durch einen einheitlichen Kanon von Methoden zusammengehalten. Fächer sind charakterisiert durch einen Set von mehr oder weniger zusammenhängenden Fragen, von mehr oder weniger zusammenängenden Problemen. Und dies, scheint mir, läst sich auch von der Philosophie sagen. Jedenfalls ist es charakteristisch für das Verständnis von Philosophie, das von vielen analytischen Philosophen geteilt wird. Bieri dagegen betont die Vergänglichkeit philosophischer Fragestellungen.

,[a] Erinnert sich noch jemand an die Probleme, mit denen Carnap im Aufbau zu kämpfen hatte, oder Goodman in The Structure of Appearance? [b] Wer weiß noch, was es alle für Wörter waren, deren Gebrauch die Schüler von Austin untersuchten, indem sie ganze Jahrgänge von Mind füllten? [c] Schreibt noch jemand über grue oder bare particulars? [d] Über die Frage, wie sich Sinnesdaten zu den Oberflächen von Dingen verhalten? [e] Ist die Unbestimmtheit der Übersetzung noch ein lebendiges Thema? [f] Oder die vielen, vielen Spielarten von Supervenienz? [g] Die Rekonstruktion des Utilitarismus in den Kategorien von Sneed? [h] Parfits Gedankenexperimente zu personaler Identität?“ (Ebd.) ${ }^{7}$

Es mag schon sein, dass es in der Philosophie Modeströmungen gibt, die sich mehr oder weniger schnell abwechseln. Aber auch das scheint mir in anderen Wissenschaften nicht anders zu sein. Und: Dem Philosophieverständnis zufolge, das, wie mir scheint, sehr viele analytische Philosophen teilen, gibt es auch eine Reihe, zeitloser' Fragen, die seit dem Beginn der Philosophie ${ }^{8}$ immer wieder gestellt wurden und die noch heute für die Philosophie kennzeichnend sind. Dazu gehören , große‘ Fragen wie:

- Gibt es einen Gott?

- Was kann man a priori wissen?

- Lässt sich das Problem der Induktion lösen?

- Worin besteht die Bedeutung sprachlicher Ausdrücke?

- In welchem Verhältnis stehen Körper und Geist zueinander?

- Haben wir einen freien Willen?

- Gibt es objektive Werte?

- Lassen sich moralische Normen rational begründen?

- Was ist eine gerechte Gesellschaft?

- Was macht eine Sache schön?

Aber auch ,kleinere` Fragen wie:

Die Antworten auf diese - natürlich nur rhetorisch gemeinten - Fragen lauten wohl: [a] Nein, weil der Phänomenalismus eine Position ist, die heute kaum noch Anhänger hat. [b] Wohl kaum noch einer. [c] Natürlich ist grue immer noch ein Thema in der Debatte um das Induktionsproblem. [d] Eher nicht, obwohl die Frage nach den direkten Objekten der Wahrnehmung immer noch virulent ist. [e] Ja, sicher. [f] Ebenfalls, obwohl inzwischen klar ist, welche Spielarten relevant sind und welche eher nicht. [g] Nein. [h] Ja, sicher.

8 Oder zumindest seit dem Zeitpunkt, zu dem diese Fragen ,entdeckt` wurden. 
Was ist im Hinblick auf sprachliche Bedeutung primär - Sätze oder Wörter?

Sind Eigennamen starre Bezeichner?

- Haben Emotionen eine kognitive Komponente?

Können Empfindungen als repräsentationale Zustände aufgefasst werden?

- Welche Rolle spielen Sinnesdaten bei der Wahrnehmung?

- Sind Farben real?

- Was spricht für den Externalismus in der Erkenntnistheorie?

- Genießen Embryonen von Anfang an den vollen Schutz der Menschenrechte?

Diese Fragen sind dem Philosophieverständnis zufolge, das ich hier im Auge habe, zeitunabhängige Sachfragen, deren Beantwortung man systematisch in Angriff nehmen kann. Philosophie ist diesem Philosophieverständnis zufolge nichts anderes als der Versuch, eben dies zu tun - der Versuch, in systematischer Weise rationale Antworten auf die Sachfragen zu finden, die das Themenspektrum der Philosophie ausmachen. Die Methode des Philosophen ist dabe neben der Begriffsanalyse in aller Regel die Analyse argumentativer Zusammenhänge. Dabe werden auch Argumente von analytischen Philosophen als etwas aufgefasst, was nicht relativ ist zu einer bestimmten Zeit, einer bestimmten Kultur oder einem philosophischen System. Bieri dagegen bezweifelt, dass es eine zeitlose Rationalität gibt. Er betont die „kontingente[ $n$ ] Randbedingungen für unser Verständnis von Vernunft“ (341), und er kritisiert die gegenteilige Auffassung:

„Frege und Russell, und später Leute wie Carnap und Hempel, gaben der analytischen Philosophie eine bestimmte Idee von Rationalität mit auf den Weg: Es war die Idee einer universellen, historisch unwandelbaren Rationalität. Das war ein cartesianisches und kantisches Erbe, und es passte perfekt zur früher besprochenen Ablehnung des Psychologismus. Es geriet mit Kuhn und dem späten Wittgenstein ins Wanken.“ (Ebd.)

Bieri bemerkt zu Recht, dass es innerhalb der Analytischen Philosophie zu diesem Thema keine einheitliche Meinung gibt. Aber mir scheint, dass die Mehrheit der analytischen Philosophen hier eher Frege, Russell, Carnap und Hempel folgt. Für sie scheint es unvernünftig, anzunehmen, dass Descartes' Gottesbeweise zu seiner Zeit ganz in Ordnung waren, für uns heute aber ihre Gültigkeit verloren haben. Es kann in ihren Augen nicht sein, dass Platon zu seiner Zeit mit seiner Ideenlehre Recht hatte, während diese Position schon für Kant nich mehr gültig war. Das heißt natürlich nicht, dass sich die Evidenzlage nicht ändern kann. Erst nachdem Russell die Paradoxien der Fregeschen Arithmetik entdeckt hatte, musste jeder - auch Frege - akzeptieren, dass mit dieser Theorie etwas nicht Ordnung war. Vorher wa Frege durchaus gerechtfertigt, sie für wahr zu halten. Unmöglich ist aber, dass es bei gleicher Evidenzlage für eine Person rational sein kann, eine Theorie für wahr zu halten, für eine andere dagegen nicht. Ein zwingendes Argument ist für alle gleich zwingend - nicht nur für die Menschen einer bestimmten Zeit oder eines bestimmten Kulturkreises. Und ein Widerspruc ist ein Widerspruch - nicht nur ein Widerspruch für die Anhänger der Transzendentalphilosophie oder der Phänomenologie.

Aus diesen Auffassungen ergibt sich ein weiteres Merkmal des von mir angesprochenen Philosophieverständnisses - die Überzeugung, dass es so etwas wie philosophische Schulen eigentlich nicht geben kann. Möglich sind nur unterschiedliche Auffassungen und Positionen aber diese sind gegeneinander nicht so abgeschottet, dass ein Austausch von Argumenten unmöglich wäre. Ganz im Gegenteil: Es gibt nur einen großen philosophischen Diskurs, in dem jeder argumentativ zu den Auffassungen der jeweils anderen Stellung nehmen kann. Dies ist auch der Grund dafür, dass Philosophie arbeitsteilig betrieben werden kann. Wenn die Aufgabe von Philosophinnen und Philosophen nicht ist, große Systeme zu entwerfen, sondern an der Klärung zeitübergreifender philosophischer Fragen mitzuwirken, dann können auch kleine Beiträge einen Fortschritt bedeuten. Sie müssen nur auf eine gemeinsame Frage bezogen sein und helfen, der Antwort auf diese Frage näher zu kommen.

Allerdings, Philosophie unterscheidet sich in einem Punkt recht deutlich von anderen Fächern: Sie kann kaum hoffen, ihre Fragen ein für alle Mal definitiv zu beantworten. Der Grund dafür ist einfach. Philosophische Fragen werden weder durch Beweise noch durch Beobachtungen oder Experimente entschieden. Vielmehr kann man sich der Beantwortung dieser Fragen nur argumentativ nähern. Alle Argumente beruhen jedoch auf Prämissen; und diese Prämissen können ihrerseits wieder in Zweifel gezogen werden. Niemand verfügt über einen archimedischen Punkt, der über jeden Zweifel erhaben wäre; niemand kann sich auf Prämissen stützen, die nicht angegriffen werden können. Das zeigt sich schon daran, dass alle angeblich sicheren Prämissen in der Geschichte der Philosophie tatsächlich irgendwann einmal bezweifelt wurden. Selbst die logischen Wahrheiten waren von diesem Schicksal nicht ausgenommen. Descartes etwa - also ein Philosoph, der wie kaum ein anderer nach einem archimedischen Punkt des Denkens gesucht hat - war der Meinung, dass die Gesetze der Logik dem Willen Gottes unterliegen. Und daraus folgt, dass wir uns selbst bei den logischen Wahrheiten irren können. Nun gibt es sicher gute Argumente, die zeigen, dass die Gesetze der Logik nicht vom Willen Gottes abhängen können. Aber natürlich beruhen auch diese Argumente auf Prämissen, die man bezweifeln kann. Selbst in diesem Punkt ist also keine definitive Entscheidung möglich.

Fortschritt besteht in der Philosophie deshalb nicht darin, einen Kanon allgemeinverbindlicher Antworten zu entwickeln; Fortschritt in der Philosophie bedeutet, dass wir die möglichen Antworten und ihre Konsequenzen besser verstehen. Es geht darum, einen möglichst umfassenden Überblick über die Antworten zu geben, die man auf eine Frage geben kann, und herauszuarbeiten, welche Implikationen diese Antworten haben, worauf man sich festgelegt hat, wenn man die eine oder die andere Antwort akzeptiert. Damit wird zugleich klar, was für und was gegen diese Antworten spricht. Da es für alle Positionen in der Philosophie Pro- und Kontraargumente gibt, wird bei diesem Vorgehen keine Antwort als die einzig mögliche oder als die einzig rationale ausgezeichnet. David Lewis hat das in der Einleitung zum ersten Band seiner Philosophical Papers sehr schön formuliert:

„Whether or not it would be nice to knock disagreeing philosophers down by sheer force of argument, it cannot be done. Philosophical theories are never refuted conclusively. (Or hardly ever. Gödel and Gettier may have done it.) The theory survives its refutation - at a price. $[\ldots]$ when all is said and done, and all the tricky arguments and distinctions and counterexamples have been discovered, presumably we will still face the question which prices are worth paying, which theories are on balance credible, which are the unacceptably counterintuitive consequences and which are the acceptably counterintuitive ones. On this question we may still differ. And if all is indeed said and done, there will be no hope of discovering still further arguments to settle our differences." (Lewis 1983, x)

Wie gesagt, diese Auffassung impliziert nicht, dass es in der Philosophie keinen Fortschritt gibt. Wenn es gelingt zu klären, was eine bestimmte Position genau impliziert, welche Argumente für diese Position relevant und welche Argumente definitiv zum Scheitern verurteilt sind, zum Beispiel weil sie nicht zeigen, was sie zeigen sollen, kann dies einen wesentlichen Fortschritt darstellen. Fortschritt in der Philosophie bedeutet im Allgemeinen nicht die 
Lösung, sondern die Klärung von Problemen. Ist es nicht das, was auch Bieri vorschwebt? Falls ja, sollte man vielleicht betonen, dass die zitierte Passage von einem der angesehensten analytischen Philosophen überhaupt stammt

Es fällt mir nicht leicht zu erkennen, was Bieri an dem gerade geschilderten Philosophieverständnis auszusetzen hat und insbesondere welche Alternative ihm vorschwebt. Nich aufgeben will er die durch die Analytische Philosophie erworbenen Standards begrifflicher Klarheit und argumentativer Rationalität:

„Beibehalten sollte man auf jeden Fall die Maßstäbe von Klarheit und gedanklicher Übersicht, welche die analytische Philosophie gesetzt hat. Sie sind von bleibendem Wert, auch im Umgang mit den Schlüsseltexten aus der Geschichte der Philosophie. Der Unterschied im Niveau zwischen Historikern, die jene Maßstäbe erfüllen, und solchen, die es nicht tun, ist gewaltig." (342)

Kein Einspruch, Euer Ehren. Aber was die Inhalte und Ziele der Philosophie angeht, fügt Bieri hinzu:

„An [...] besseren Tagen würde ich sagen: [Analytische Philosophie] hat sehr wohl eine Zukunft. Ich sehe sie im Projekt einer deskriptiven Metaphysik der Erfahrung, die sich die vielen sprachanalytischen Einsichten dieser Philosophie zu Eigen macht, ohne sich irgendwelchen Dogmen zu verschreiben, weder quineanischen noch wittgensteinianischen. Man könnte das Projekt auch in kantischen Worten beschreiben: Orientierung im Denken. Eine Orientierung, in der wir uns vergewissern, wie wir über uns und unsere Erfahrung der Welt denken und vielleicht denken müssen, wenn es eine verständliche Welt sein soll. [...] Die Orientierung im Denken, die man auf diese Weise erwirbt, ist manchmal auch von praktischer Bedeutung, etwa bei moralphilosophischen Themen. Doch darum geht es mir nur am Rande. Wichtiger ist mir die gedankliche Übersicht als solche. Wenn man sie gewonnen hat, ist man ein anderer Mensch als vorher." (343)

Ist die Übersicht, die Bieri vorschwebt, eine andere als die, über die ich gerade gesprochen habe? Was genau ist damit gemeint, dass ,wir uns vergewissern, wie wir über uns und unsere Erfahrung der Welt denken und vielleicht denken müssen, wenn es eine verständliche Wel sein soll“"? Gehört dazu, dass wir nach wie vor versuchen, die traditionellen Fragen der Philosophie zu beantworten, oder geht es um ganz andere Fragen? Was genau sind die Probleme, mit denen sich Bieri zufolge die Philosophie beschäftigen sollte? Und warum sollte es ein Zie der Philosophie sein, dass man durch die Beschäftigung mit ihr ein anderer Mensch wird? Schon relativ früh in seinem Aufsatz findet sich die Passage:

„Stellen wir uns vor, wir verbringen einen Monat damit, vormittags das Journal of Philosophy zu lesen und nachmittags die Werke von Seneca, Montaigne, Nietzsche, Cesare Pavese und Fernando Pessoa. Dann bekommen wir ein Gefühl dafür, dass mit der Professionalisierung der Philosophie durch die analytischen Autoren etwas ganz Neues geschehen ist und dass es nicht selbstverständlich ist." $(334)^{9}$

9 Hanjo Glock hat ein interessantes Spiel vorgeschlagen, dessen Pointe die Frage ist, welche Namen man in Bieris Aufzählung durch welche anderen ersetzen kann (Glock 2008,9). Kann man die zweite Gruppe von Namen ersetzen durch „Plotin, Vico, Hamann, Schelling und Hegel“" oder durch "Heidegger, Derrida, Irigaray, Deleuze und Kristeva"? In diesem Sinne könnte man auch fragen, ob das „Neue“, das nach Bieri erst durch die Analytische Philosophie ins Spiel gekommen ist, nicht
Auch hier könnte es um unterschiedliche Fragen gehen, aber auch darum, dass Bieri sich eine andere Art wünscht, mit diesen Fragen umzugehen. Möchte er, dass die Philosophie essayistischer wird und aufhört, systematisch nach begründeten Antworten zu suchen? Auf jeden Fall pflichtet er Rorty in der Auffassung bei,

„dass philosophischer Fortschritt nicht im geduldigen Verfolgen von kleinteiligen Forschungsprojekten einer universell arbeitenden Vernunft besteht, sondern in überraschenden

Leistungen unserer durch und durch kontigenten, idiosynkratischen Einbildungskraft" (343).

Ich bin da ganz anderer Meinung. Dabei soll die Bedeutung der Leistungen der Einbildungskraft keineswegs geschmälert werden. Keine Wissenschaft kann ohne sie auskommen. Alle wirklich neuen Ideen (siehe Newton, siehe Einstein) beruhen auf der Fähigkeit, die Dinge einmal völlig neu und anders zu sehen. Aber bedeutet das, dass das geduldige Verfolgen von kleinteiligen Forschungsprojekten nicht auch viel zum philosophischen Fortschritt beiträgt, für diesen Fortschritt sogar unerlässlich ist?

Es gibt so etwas wie philosophische Essayistik - ein geistreiches Schreiben über dies und das, ohne dass man den Eindruck hätte, dass sich hier jemand systematisch bemühen würde, ein sachliches Problem von allen Seiten zu beleuchten und so einer Lösung näher zu bringen. Man vergleiche etwa Texte von Kant und Montaigne. Diese Art des philosophischen Schreibens hat durchaus ihre Berechtigung - nicht nur dann, wenn sie den Leser unterhält (das kann man von Kants Kritik der reinen Vernunft sicher nicht erwarten), und vielleicht sogar erbaut, sondern besonders dann, wenn sie Unordnung in eine festgefahrene Diskussion bringt und so neue Sichtweisen auf schon bekannte Probleme ermöglicht. Aber philosophische Essayistik kann nie den Kern des philosophischen Tuns ausmachen. Jedenfalls dann nicht, wenn man dieses Tun als den systematischen Versuch auffasst, rationale Antworten auf Sachfragen zu erarbeiten. ${ }^{10}$ Dies geht auf die Dauer nur in systematischen Abhandlungen, die - das zeigt sich auch in anderen Wissenschaften - in der Regel eher eine trockene Kost sind.

Hanjo Glock zitiert in seinem Buch What is Analytical Philosophy? Hao Wang, der Analytische Philosophie als ,wissenschaftszentriert“ charakterisiert und von der „kunstzentrierten“ Philosophie Wittgensteins abgrenzt:

„Unlike Russell, Carnap and Quine, Wittgenstein is art centered rather than science centered and seems to have a different underlying motive for his study of philosophy." (Wang 1986, 75)

Manchmal denke ich, dass es Bieri ähnlich sieht - dass er eine kunstzentrierte Philosophie anstrebt und die wissenschaftszentrierte Philosophie sehr vieler analytischer Philosophen ablehnt. Seine Vorliebe für Wittgenstein und den normalsprachlichen Zweig der Analytischen Philosophie sind ja deutlich genug.

doch schon früher vorhanden war. Kann man zum Beispiel „das Journal of Philosophy“ ersetzen durch „die Werke Aristoteles', Descartes' und Kants“?

10 „Systematisch“ heißt hier natürlich nicht, dass es um die Errichtung großer Systeme ginge, sondern um ein geordnetes und kontrolliertes Vorgehen, bei dem man Schritt für Schritt und möglichst, ohne etwas zu übersehen, versucht, seinem Ziel näher zu kommen. 


\section{VI.}

Was kann „Analytische Philosophie“ heute noch heißen? Glock hat in seinem Buch What is Analytical Philosophy? eine große Zahl von Vorschlägen, den Begriff ,Analytische Philosophie" zu präzisieren, gründlich und ausführlich untersucht. Sein Ergebnis ist im Wesentlichen negativ: Alle Versuche, eines oder mehrere Merkmale anzugeben, die genau den Philosophinnen und Philosophen gemeinsam sind, die zur Analytischen Philosophie gerechnet werden müssen, sind gescheitert. ${ }^{11}$ Angesichts dieses Befundes schlägt Glock letzten Endes vor, „Analytische Philosophie“ als einen durch Familienähnlichkeiten charakterisierten Begriff zu verstehen.

„The first is the idea that analytic philosophy should be explained in terms of family resemblances. What holds analytic philosophers together is not a single set of necessary and sufficient conditions, but a thread of overlapping similarities (doctrinal, methodological and stylistic). Thus current analytic philosophers may be tied to Frege and Russell in their logical methods, or to logical positivism and Quine in their respect for science, or to Wittgenstein and linguistic philosophy in their concern with the a priori, meaning and concepts, etc.“ (Glock 2008, 19)

,[A]nalytic philosophy is a historical tradition held together by ties of influence on the one hand, family resemblances on the other." (Glock 2008, 231)

Das ist ein sehr interessanter Vorschlag. Aber ist es wirklich wichtig, eine Antwort auf die Frage „Was ist Analytische Philosophie?“ zu finden? Ich muss gestehen, dass mich diese Antwort weniger und weniger interessiert. Da geht es mir wie Peter Bieri. Auch ich möchte viel eher herausfinden, was gute Philosophie ist und wie man diese Philosophie betreiben sollte. ${ }^{12}$ Meine Antworten sind dabei aber ganz andere als die, von denen ich vermute, dass sie Bieri gefallen würden. Besonders zwei Dinge sind mir wichtig:

1. Die Auffassung, dass es in der Philosophie darum geht, in systematischer Weise rationale Antworten auf die Sachfragen zu finden, die das Themenspektrum der Philosophie ausmachen; dass es dabei Standards der Rationalität gibt, die für alle in gleicher Weise gelten; und dass es deshalb letzten Endes nur einen großen philosophischen Diskurs geben kann.

2. Die Auffassung, dass die Arbeit der Philosophie nur dann erfolgversprechend geleistet werden kann, wenn man versucht, die verwendeten Begriffe in all ihren möglichen Lesarten so klar und argumentative Zusammenhänge so transparent wie möglich zu machen, wobe Ergebnisse der modernen Logik überall da zu berücksichtigen sind, wo es der Sache dient.

11 Neben vielem anderen geht Glock auch auf die von mir behandelten Merkmale „Metaphysikkritik“ „Wissenschaftsnähe“, „Naturalismus“ und „,besondere Betonung der Logik“ ein und führt penibel auf, welche der gemeinhin als analytische Philosophinnen und Philosophen geltenden Personen diese Merkmale erfüllen und welche nicht. Auch bei diesen Merkmalen gilt, dass keines auf alle zutrifft.

12 Es gibt eine hübsche Anekdote über den Gründungskongress der Gesellschaft für Analytische Philosophie. Im ersten Entwurf der Satzung war vorgesehen, das, was Analytische Philosophie ausmacht, sophie. Im ersten Entwurf der Satzung war vorgesehen, das, was Analytische Philosophie ausmacht,
zumindest kurz zu charakterisieren: Analytische Philosophie ist in besonderer Weise begriffliche zumindest kurz zu charakterisieren: Analytische Philosophie ist in besonderer Weise begriffliche
Klarheit und argumentativer Rationalität verpflichtet. Daraufhin ging Friedrich Kambartel an Klarheit und argumentativer Rationalitat verpflichtet. Daraufhin ging Friedrich Kambartel an Rednerpult und sagte, er freue sich, dass man offenkundig gewillt sei, eine Gesellschaft für Erlan-
ger/Konstanzer Philosophie zu gründen; denn schließlich seien es die Erlanger und Konstanzer, ger/Konstanzer Philosophie zu grunden; denn schließlich seien es die Erlanger und Konstanzer,
denen diese Merkmale besonders wichtig seien. Gelächter und Gemurmel im Saal: Vielleicht solle man doch lieber gleich eine Gesellschaft für gute Philosophie gründen.
Es gibt inzwischen hervorragende Einführungen in die verschiedenen Bereiche der Philosophie - zum Beispiel das Buch Philosophy of Religion: An Introduction von William Rowe, in dem diese beiden Aspekte guter Philosophie auf geradezu hervorragende Weise verwirklicht und miteinander verbunden sind. Meiner Meinung nach kann man Philosophie nicht besser betreiben. Und deshalb wünsche ich mir natürlich, dass diese Art des Philosophierens noch sehr viel mehr Anhänger gewinnt, als sie heute schon hat.

Prof. Dr. Ansgar Beckermann, Universität Bielefeld, Fakultät für Geschichtswissenschaft, Philosophie und Theologie, Abteilung Philosophie, Postfach 1001 31, 33501 Bielefeld

\section{Literatur}

Beckermann, A. (2004), Einleitung, in: P. Prechtl (Hg.), Grundbegriffe der Analytischen Philosophie, Stuttgart, 1-12.

Bieri, P. (2007), Was bleibt von der Analytischen Philosophie?, in: Deutsche Zeitschrift für Philosophie, Jg. 55, Heft 3, 333-344.

Carnap, R. (1928), Scheinprobleme in der Philosophie. Nachwort von Günther Patzig, Frankfurt/M. 1966; 1. Aufl., Berlin 1928.

Carnap, R. (1931), Überwindung der Metaphysik durch logische Analyse der Sprache, in: Erkenntnis, 2; Wiederabdruck in: H. Schleichert (Hg.), Logischer Empirismus - der Wiener Kreis, München $1975,149-171$.

Carnap, R.; Hahn H.; Neurath, O. (1929), Wissenschaftliche Weltauffassung - der Wiener Kreis. Wien: Veröffentlichungen des Vereins Ernst Mach; Wiederabdruck in: H. Schleichert (Hg.), Logischer Empirismus - der Wiener Kreis, München 1975, 201-222.

Fellmann, F. (1998), Orientierung Philosophie. Was sie kann, was sie will, Reinbek bei Hamburg. Geyer, Ch. (2007), Sorge dich nicht, denke, in: Frankfurter Allgemeine Zeitung, 19. September 2007 Glock, H. J. (1996), A Wittgenstein Dictionary, Oxford.

Glock, H. J. (2008), What is Analytic Philosophy?, Cambridge.

Kim, J. (2003), The American Origins of Philosophical Naturalism, in: Journal of Philosophical Research, 83-98.

Lewis, D. (2001), Counterfactuals, 2., überarb. Auff., Oxford.

Lewis, D. (1983), Introduction, in: ders., Philosophical Papers, Bd. 1, Oxford, ix-xii.

Lewis, D. (1991), Parts of Classes, Oxford

Quine, W. V. O. (1951), Two Dogmas of Empiricism, in: ders., From a logical point of view, 3. Aufl., New York 1961.

Rowe, W. (2001), Philosophy of Religion - An Introduction, 3. Aufl., Wadsworth.

Schwarz, W. (2008), David Lewis: Metaphysik und Analyse, Diss. phil., Bielefeld.

Sellars, W. F. (1963), Science, Perception and Reality, London.

Strawson, P. F. (1959), Individuals, London.

Wang, H. (1986), Beyond Analytic Philosophy, Cambridge/Mass

Wittgenstein, L. (1921), Tractatus logico-philosophicus; Wiederabdruck in: ders., Schriften, Bd. 1, Frankfurt/M. 1969, 7-83. 